\title{
Determinación del potencial de los recursos hídricos subterráneos de la región Ica
}

\author{
Carlos Sebastián Calvo \\ Universidad Ricardo Palma \\ carlos.sebastianc @urp.pe
}

\begin{abstract}
RESUMEN
La región de Ica-Perú, tiene un gran potencial agroexportador en el país. Es una región privilegiada para este tipo de inversiones por su calidad de suelo, clima, horas de sol y luminosidad, lo que la hace muy competitiva y con altos índices de productividad y de calidad en sus cosechas. El empleo en Ica creció a un nivel del $31 \%$ mensual durante los periodos de cosecha dedicados a la agro exportación, logrando el pleno empleo, lo que le ha permitido que su población pueda acceder a mejores niveles de vida y consumo. Se ha identificado como el problema central, la sobreexplotación de los acuíferos del área del proyecto; evidenciado por el descenso continuo de los niveles de la napa freática, hecho que viene afectando reservas de agua subterránea y, en algunos casos, la calidad de las mismas. Además, el irracional y deficiente aprovechamiento del agua subterránea tiene como causas indirectas la informalidad en la explotación del acuífero.
\end{abstract}

Palabras clave: Agua subterránea, acuífero, hidrogeoquímica, análisis isotópico, modelamiento hidráulico

\section{Potential ascertainment of underground water resources in Ica region}

\begin{abstract}
The Ica-Peru region has great agro-export potential in the country. It is a privileged region for this type of investment because of its quality of soil, climate, hours of sunshine and luminosity, which makes it very competitive; achieving high levels of productivity and quality in their crops. Employment in Ica grew at a level of $31 \%$ per month during the harvest periods dedicated to agricultural exports, achieving full employment, which has allowed its population to access higher levels of living and consumption.

The overexploitation of aquifers in the project area has been identified as the central problem evidenced by the continuous descent of groundwater levels, a fact that is affecting groundwater reserves, and in some cases, the quality of them. In addition, the irrational and deficient use of groundwater has as indirect causes the informality in the exploitation of the aquifer.
\end{abstract}

KeYwORDs: Groundwater, aquifer, hydro geochemistry, isotopic analysis, hydraulic modeling 


\section{Introducción}

Las informaciones basadas en el uso de técnicas analíticas químicas y de isótopos ambientales presentes en el agua y en las sustancias disueltas proporcionan información única sobre el origen, flujos y la historia de los cuerpos de agua, destacándose las siguientes aplicaciones:

- Evaluación de aguas subterráneas (origen, dinámica, flujo, transporte, datación, recarga, calidad del agua, causas naturales vs. impacto de actividades humanas, etc.).

- Aguas superficiales (hidrología de cuencas hidrológicas, flujo de base de los ríos, interacción entre aguas subterráneas y ríos/lagos/humedales).

- Temas multisectoriales (gestión de las aguas subterráneas, uso conjuntivo de aguas superficiales y subterráneas, gestión de cuencas, sistemas transfronterizos, zonas costeras, humedales, etc.)

Se ha establecido que en la zona de estudios existe un creciente déficit de abastecimiento debido a los siguientes motivos:

- Incremento de la demanda por el crecimiento de la población.

- Incremento de la producción agropecuaria e industrial.

- Sobreexplotación de aguas superficiales y subterráneas conocidas

- Desconocimiento del potencial de aguas subterráneas.

- Reducción de la calidad de las fuentes de agua debido a la contaminación de aguas superficiales y subterráneas por el vertimiento de desechos urbanos, industriales y agrícolas.

- Ausencia de tecnologías de recarga inducida de aguas subterráneas.

La información obtenida con técnicas hidrogeoquímicas e isotópicas proporciona las bases científicas para la adopción de medidas encaminadas a mejorar la gestión de los recursos hídricos (en cantidad, calidad y sostenibilidad).

\section{Antecedentes}

En el valle de Ica y en las Pampas de Villacurí y Lanchas, se han realizado en los últimos quince años varios estudios a cargo de entidades públicas y privadas que guardan relación con el presente estudio, destacándose los siguientes:

- Inventario y evaluación de las fuentes de agua subterránea del valle del río Ica (Villacurí).

- Plan de gestión de la oferta de agua en las cuencas del ámbito del Proyecto Tambo-Ccaracocha. 
- Estudio hidrogeológico del valle Ica (Villacurí) y estado actual de las fuentes de agua subterránea, proporcionando información sobre la masa explotada del acuífero.

- Diagnóstico de la oferta hídrica de los acuíferos del valle de Ica y Pampas de Villacurí, mediante modelación numérica para determinar la oferta hídrica subteránea de los acuíferos de Ica y Pampas de Villacurí.

- Modelación matemática del sistema acuífero Ica-Villacurí; programa de formalización de los derechos de uso del agua.

- Modelo del acuífero de Ica-Villacurî" (modelamiento numérico de flujo del valle de Ica y Pampas de Villacurí, empleando el software Visual Modflow). La simulación la realiza en régimen estacionario y transitorio.

- Informe sobre pruebas de infiltración. El estudio busca evaluar la infiltración con fines de recargar el acuífero de Villacurí mediante embalses de Infiltración.

- Inventario de fuentes de agua subterránea Pampas de Lanchas.

- Plan de gestión específico para los acuíferos de Ica, Villacurí y Lanchas, con la finalidad de contribuir a la solución del problema de sobreexplotación.

\section{El problema de sobreexplotación de los acuíferos del departamento de Ica}

La sobreexplotación de los acuíferos se ha evidenciado por el descenso continuo de los niveles de la napa freática, hecho que viene afectando reservas permanentes (no renovables) de agua subterránea y, en algunos casos, la calidad de las mismas como en los acuíferos de Villacurí.

Además de la causa directa antes indicada, el irracional y deficiente aprovechamiento del agua subterránea tiene como causas indirectas: la informalidad en la explotación del acuífero, el incumplimiento de las normas sobre la materia, insuficiente personal especializado que devino el deficiente control y vigilancia de la explotación de los acuíferos y la falta de la instalación de instrumentos de medición del consumo del agua subterránea.

El incremento de la demanda se ha originado por la agricultura de agroexportación con cultivos de alto consumo de agua, la ampliación de la frontera agrícola, la escasez o inexistencia de agua superficial para equilibrar la demanda de la agricultura con el uso conjunto.

Finalmente, se ha identificado como causa indirecta el insuficiente conocimiento sobre la gestión del agua subterránea debido a la escasa información.

Es necesario contribuir con propuestas técnicas para incrementar la oferta de agua subterránea del departamento de Ica, mediante estudios hidrogeoquímicos y técnicas isotópicas. 


\section{Zona de influencia}

Comprende la cuenca integral del río Ica y tiene un área total de $8103 \mathrm{~km}^{2}$ (nacientes del río Pampas, hasta la desembocadura del río Ica en el Pacífico), que incluye el sistema Choclococha, con un área total: $392 \mathrm{~km}^{2}$ y con una altitud media de 4600 m.s.n.m., y la cuenca natural del río Ica, con un área total $7711 \mathrm{~km}^{2}$ y con una altitud de 0,00 4503 m.s.n.m. La figura 1 muestra la conexión de las diferentes fuentes del sistema bajo investigación.

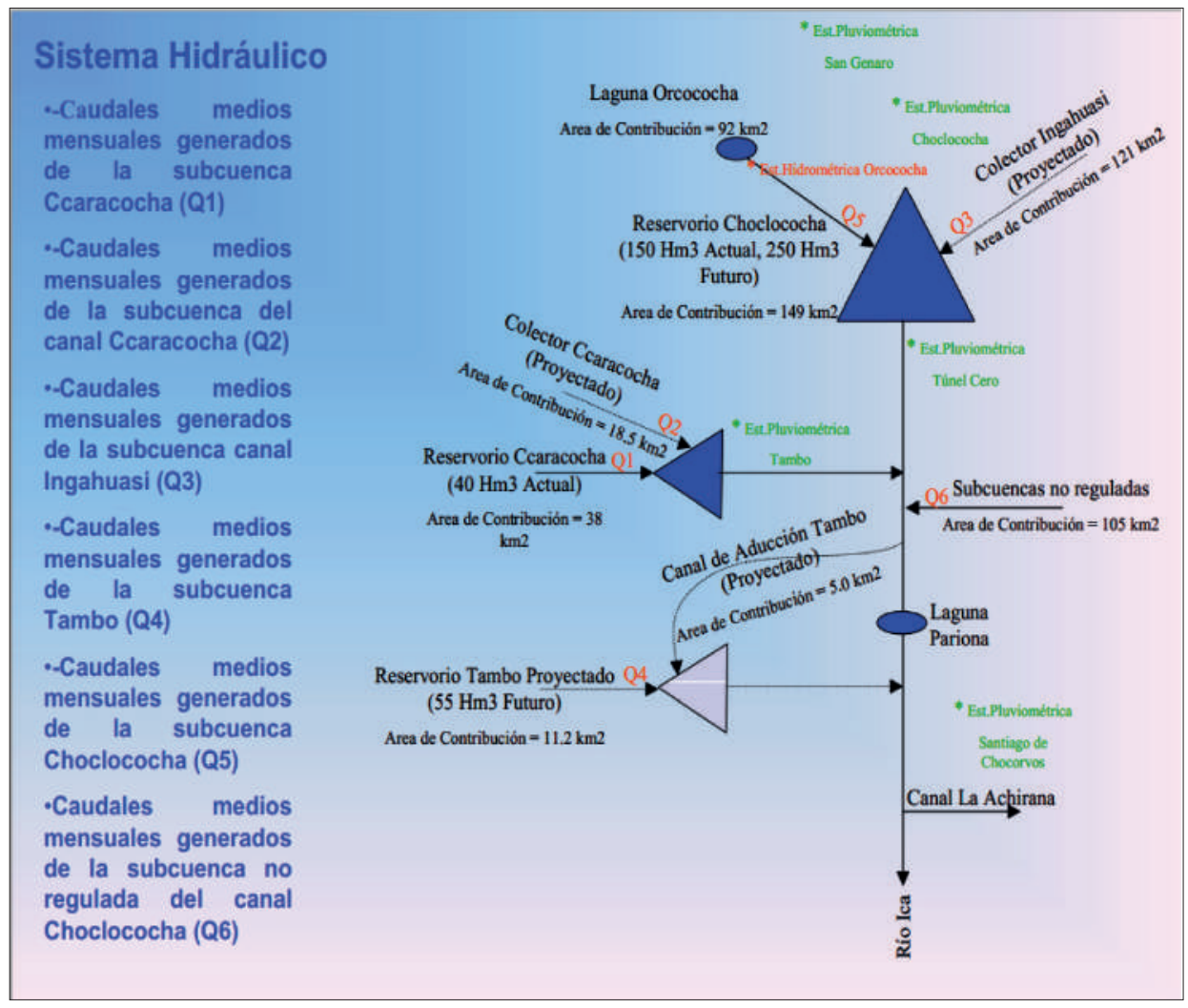

Figura 1. Diagrama de conexiones del área de influencia de los acuiferos de la Región Ica

Los acuíferos Ica, Villacurí y Lanchas se encuentran ubicados en la costa central del Perú, aproximadamente entre los km. 239 y 340 de la carretera Panamericana Sur.

El acuífero Pampa Lanchas se encuentra ubicado en la provincia de Pisco y departamento de Ica; comprende los distritos de Paracas y parte de los distritos de Humay, San Andrés y Túpac Amaru, que se encuentran sobre la margen izquierda del río Pisco. 
El área total, cuya extensión superficial es de $5100 \mathrm{~km}^{2}$. Se encuentra delimitado por el polígono determinado de vértices 1-2-3-4-5-6-7-8, tal como se muestra en el plano de la figura 2.

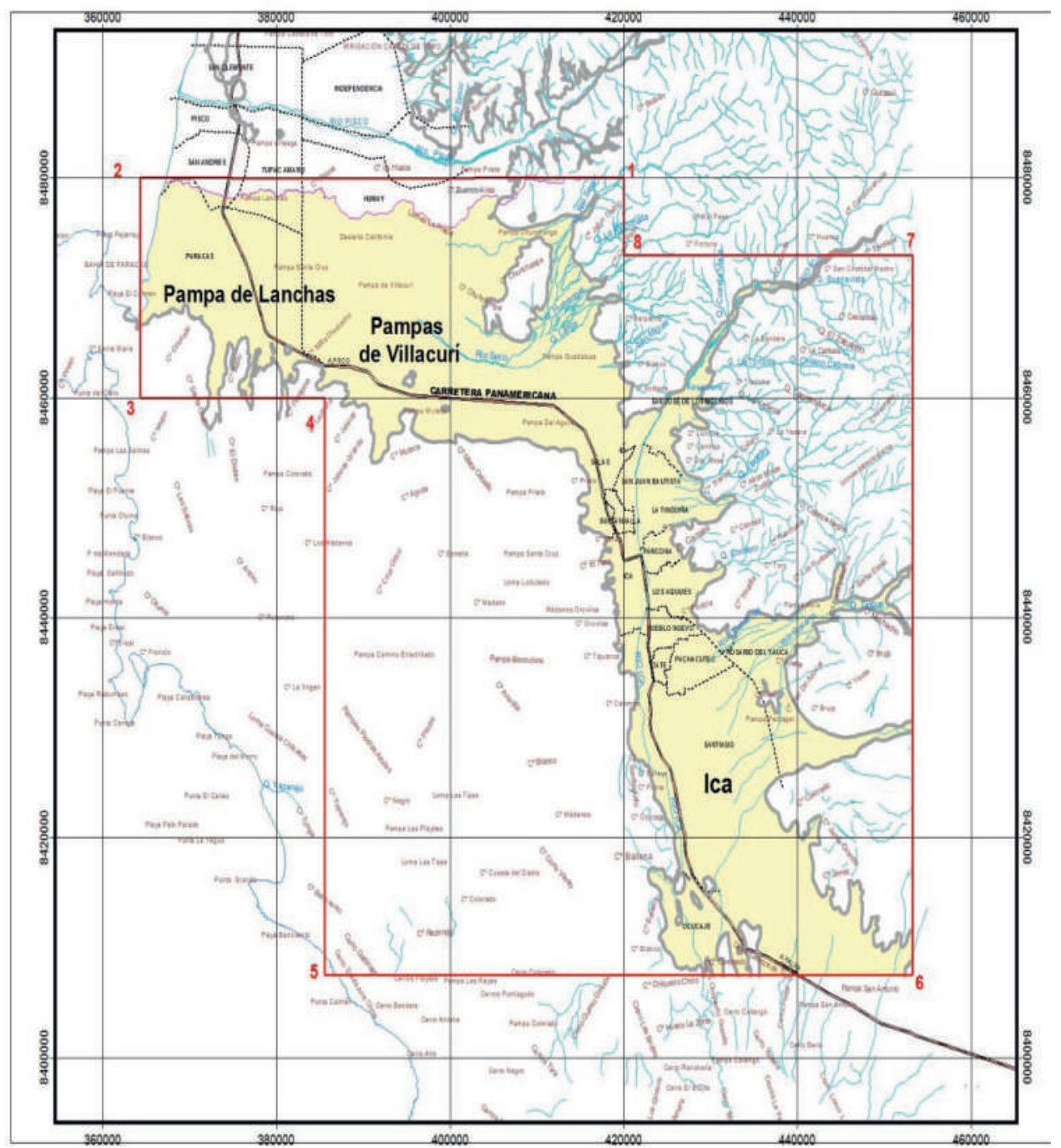

Figura 2. Localización de los acuiferos del valle de Ica y de Pampas de Villacuri y Lanchas

Dentro del referido polígono de veda se encuentran comprendidos los acuíferos del valle de Ica, así como de las Pampas de Villacurí y de Lanchas con una extensión superficial total de $1765,92 \mathrm{~km}^{2}$. 


\section{Caracterización hidrogeológica}

\subsection{Los reservorios acuíferos Ica y Villacurí}

El reservorio acuífero del valle de Ica está constituido por sedimentos fluvio-aluviales no consolidados de edad Cuaternaria, depositados, esencialmente, por el río Ica. Está conformado, principalmente, por depósitos aluviales y en forma secundaria por coluviales, siendo el primero de los nombrados el más importante para la prospección y explotación de las aguas subterráneas. Litológicamente está constituido por cantos, gravas, guijarros, arenas, arcillas y limos entremezclados en diferentes proporciones, formando horizontes de espesores variables, los mismos que se presentan en forma alternada en sentido vertical.

La prospección geofísica y la geología han determinado en el valle de Ica que los depósitos aluviales cubren el lecho rocoso, impermeable en la parte superior del valle (sector Trapiche), observándose que, aguas abajo, los aluviales suprayacen a los clastos finos (arcillosos), impermeables con potencias mayores de $250 \mathrm{~m}$. La profundidad del lecho rocoso se encuentra entre $60 \mathrm{~m}$ y más de $600 \mathrm{~m}$ de profundidad; sin embargo, no todo el espesor es acuífero aprovechable, dado que sobre el fondo del lecho rocoso se encuentran acumulados sedimentos finos e impermeables correspondientes a la formación Pisco, que pueden llegar hasta más de $350 \mathrm{~m}$ de espesor. El espesor total del acuífero aprovechable en el valle de Ica y Pampas de Villacurí varía entre 60 y 240 m, mientras que el espesor saturado entre 20 y $190 \mathrm{~m}$.

La napa en el valle de Ica es predominantemente libre, siendo su fuente de alimentación las aguas que se infiltran de la parte alta de la cuenca (zona húmeda), así como también las que se infiltran a través del lecho del río Ica, de los canales no revestidos, de regadío y en las áreas de cultivo que se encuentran bajo riego. También son fuentes de recarga del acuífero las aguas que provienen de las quebradas.

En Villacurí, la napa es predominantemente libre, aunque a mayor profundidad y en ciertos sectores podría haber confinamiento o semiconfinamiento. $\mathrm{Su}$ fuente principal de alimentación

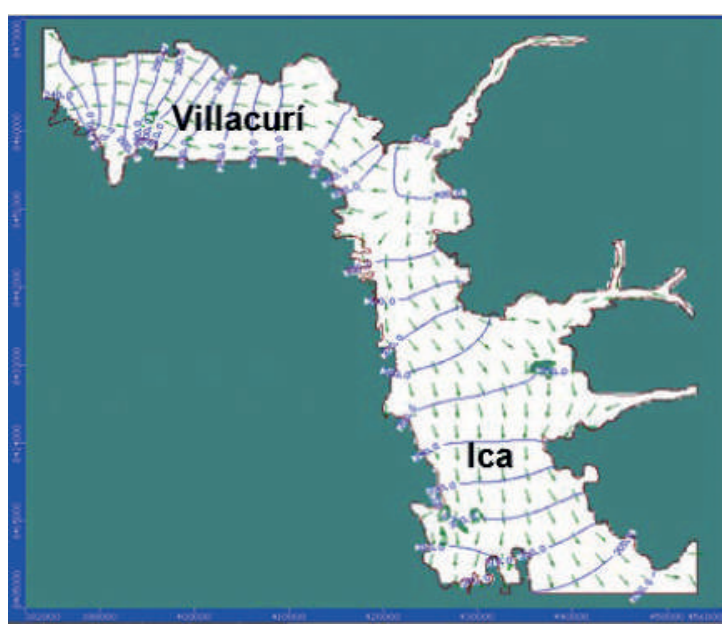

Figura 3. Representación de hidroisohipsas obtenidas en la calibración del modelo de simulación del acuifero Villacurí. 
es el flujo subterráneo proveniente del valle de Ica, aproximadamente a la altura de la curva hidroisohipsa, a $390 \mathrm{msnm}$, donde existe una interconexión hidráulica, a través de la cual fluye hacia el acuífero de Villacurí un caudal de $2 \mathrm{~m}^{3} / \mathrm{s}$, determinado por simulación numérica del sistema acuífero.

En la figura 3 se muestra el mapa de hidroisohipsas obtenido en la calibración del modelo de simulación del acuífero, en donde se puede observar los sentidos del flujo subterráneo y las curvas hidroisohipsas, que en el caso del valle de Ica van de norte a sur, de 400 a 250 msnm, mientras que en Villacurí varía de este a oeste, de 390 hasta $240 \mathrm{msnm}$.

En general, el nivel de la napa en el valle de Ica fluctúa estacional y plurianualmente, pero con una tendencia al constante descenso, como consecuencia del incremento progresivo de la explotación, evidenciando el estado de desequilibrio en que se encuentra el sistema acuífero.

\subsection{El reservorio acuífero Pampas de Lanchas}

El acuífero de la Pampa de Lanchas está conformado por depósitos aluviales, de unos 40 $\mathrm{m}$ de espesor, constituidos esencialmente por gravas y arenas de la antigua terraza del río Pisco, muy mezcladas con arcillas. En este estrato acuífero yace la formación Pisco del Terciario, constituida por arenisca finamente estratificada, diatomitas y arcilla, con yeso y ceniza volcánica impermeable que se extiende hasta alcanzar el lecho rocoso que se encuentra a unos $600 \mathrm{~m}$ de profundidad. El acuífero se alimenta a través de las filtraciones del río Pisco, de donde las aguas subterráneas fluyen de este a oeste hasta el Océano Pacífico. Del sureste llegan a la Pampa de Lanchas, excedentes de agua subterránea de la Pampa de Villacurí a través de una sección estrecha del acuífero.

En proximidades del río Pisco, la profundidad del nivel de la napa varía de 3 a $5 \mathrm{~m}$, disminuyendo hasta 0 , en donde el nivel de la napa llega a aflorar debido a la presencia de la formación Pisco, constituida por sedimentos finos e impermeables, por lo que el nivel de la napa se eleva al represarse el flujo subterráneo. Aguas abajo, ya en el distrito de Pisco, la profundidad del nivel de la napa aumenta desde 0 hasta $5 \mathrm{~m}$.

\section{Metodología}

Para establecer la metodología y la interpretación de los resultados, se tomó en cuenta la información de geología y litología del acuífero, de prospecciones geofísicas, entre otras.

El estudio se ha realizado en etapas que incluyen: a) Diseño y definición de la red optimizada para el monitoreo isotópico y físico-químico en el agua subterránea y superficial, b) La caracterización isotópica y físico-química de las aguas y las precipitaciones 
en la zona de estudio, y c) Estudios complementarios de geología y litología del acuífero, hidrodinámica, hidrogeoquímica y modelamiento.

La aplicación de técnicas isotópicas se realizó mediante análisis de concentraciones de isótopos ambientales estables deuterio $\left({ }^{2} \mathrm{H}\right)$, oxígeno $18\left({ }^{18} \mathrm{O}\right)$, isótopos radioactivos ambientales como el tritio $\left({ }^{3} \mathrm{H}\right)$ e isótopos ambientales carbono $13\left({ }^{13} \mathrm{C}\right)$ y carbono $14\left({ }^{14} \mathrm{C}\right)$, en muestras de aguas subterráneas del acuífero y de aguas superficiales de las cuencas altas de los ríos Ica y Pisco.

\section{Resultados obtenidos}

a. Se colectaron muestras para el análisis químico e isotópico por ${ }^{18} \mathrm{O},{ }^{2} \mathrm{H} \mathrm{y}{ }^{3} \mathrm{H}$, (oxígeno-18, deuterio y tritio) en zonas de trasvase y manantiales de las localidades de la zona alta de la cuenca del río Ica. En el valle de Ica, se realizó la toma de muestras teniendo en cuenta que existen plantas de tratamiento de agua residual, algunas de las cuales han colapsado con posible contaminación del acuífero. Las figuras 4 y 5 , muestran la tendencia de los resultados analíticos de $\delta \mathrm{O}-18$ y $\delta \mathrm{H}-2$.

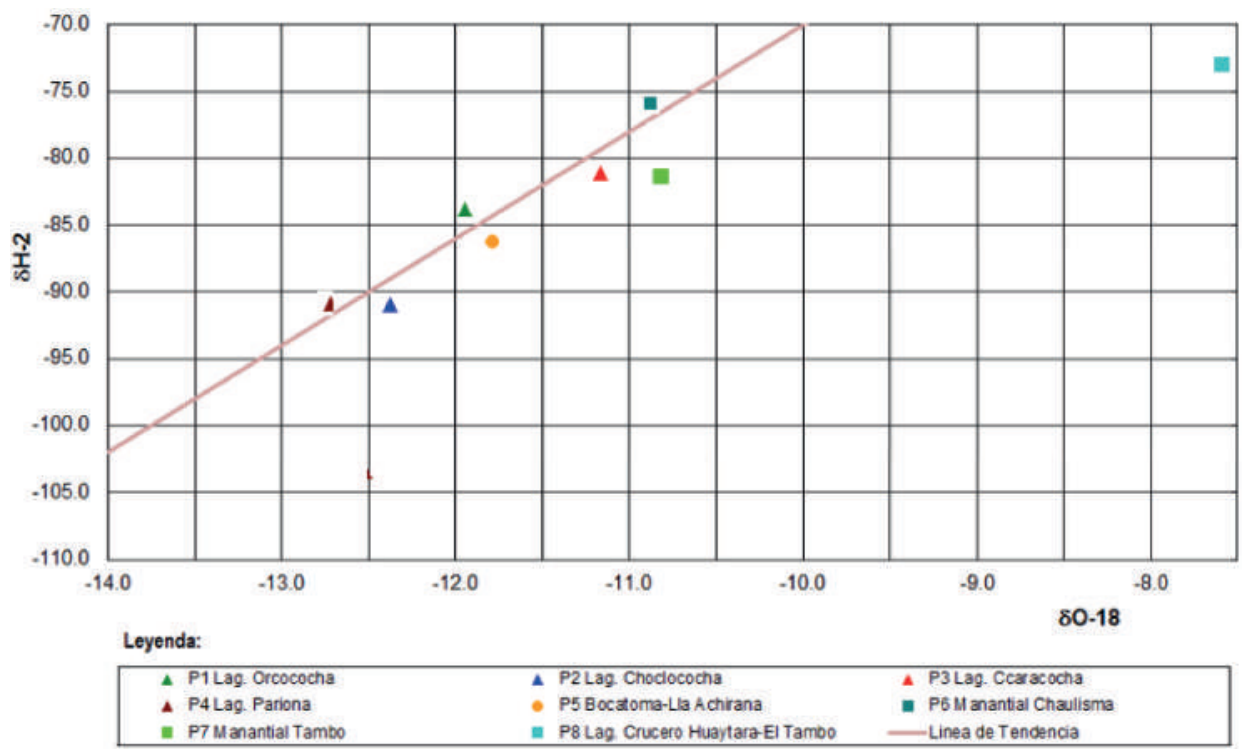

Figura 4. Análisis de $\delta \mathrm{O}-18$ y $\delta \mathrm{H}-2-$ Zona de trasvase (lagunas) y río Ica. 

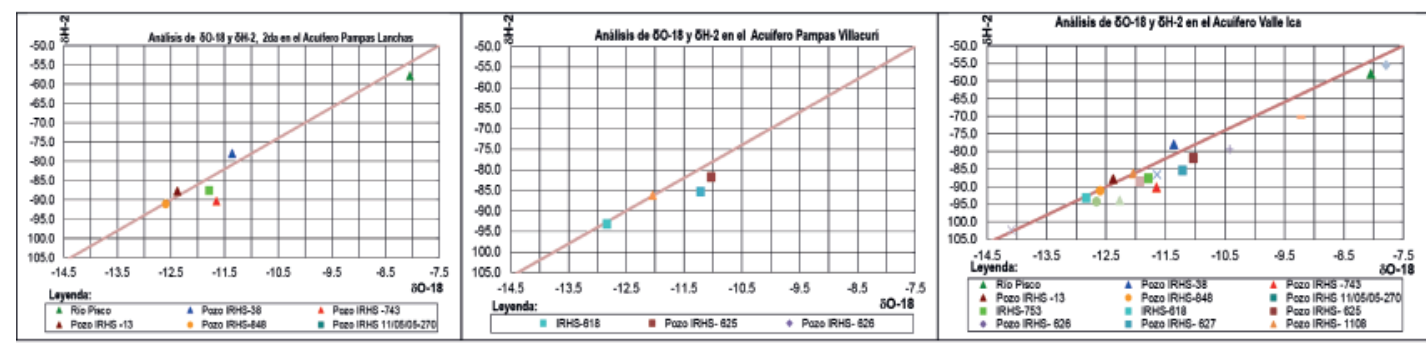

Figura 5. Resultados de análisis de $\delta \mathrm{O}-18$ y $\delta \mathrm{H}-2$ de los acuiferos Valle Ica, Pampas Villacurí y Lanchas.

c. Se realizó el muestreo para análisis isotópico de los acuíferos de las Pampas de Villacurí y Lanchas. En las figuras 6 y 7, se aprecian los resultados isotópicos de los puntos monitoreados.

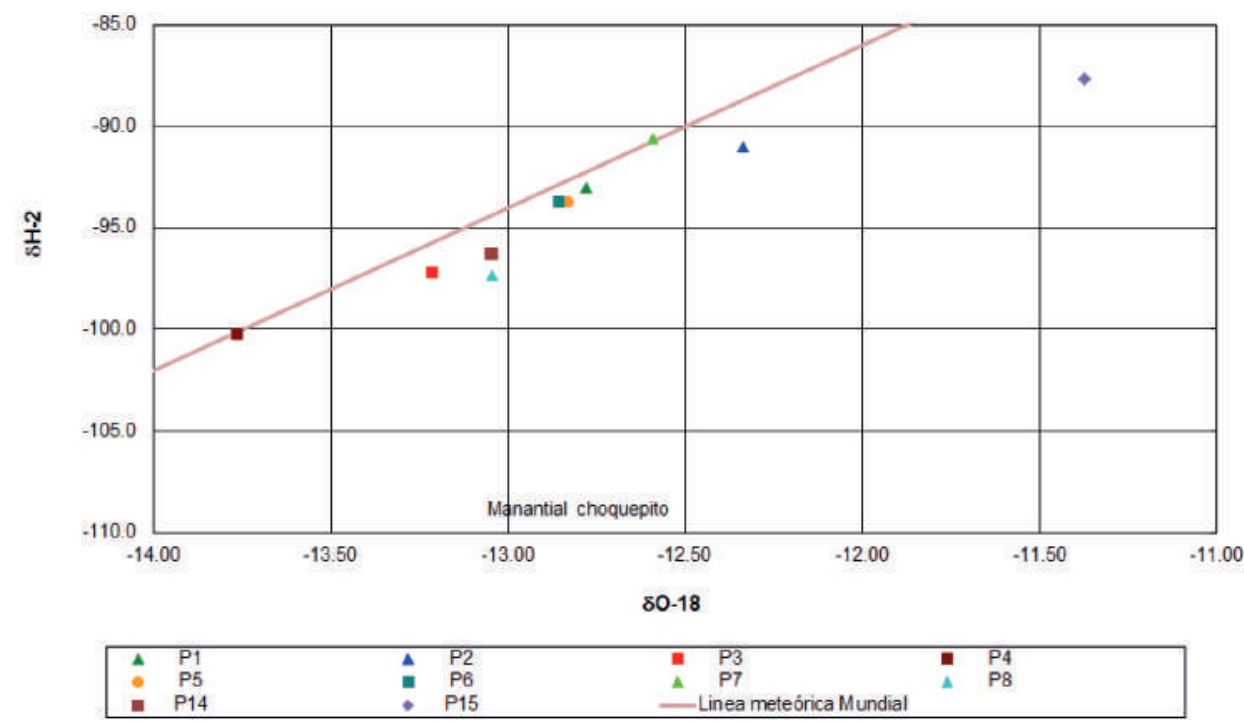

Figura 6. Análisis de $\delta O-18$ y $\delta H-2$ - Acuifero Pampas Villacurí. 


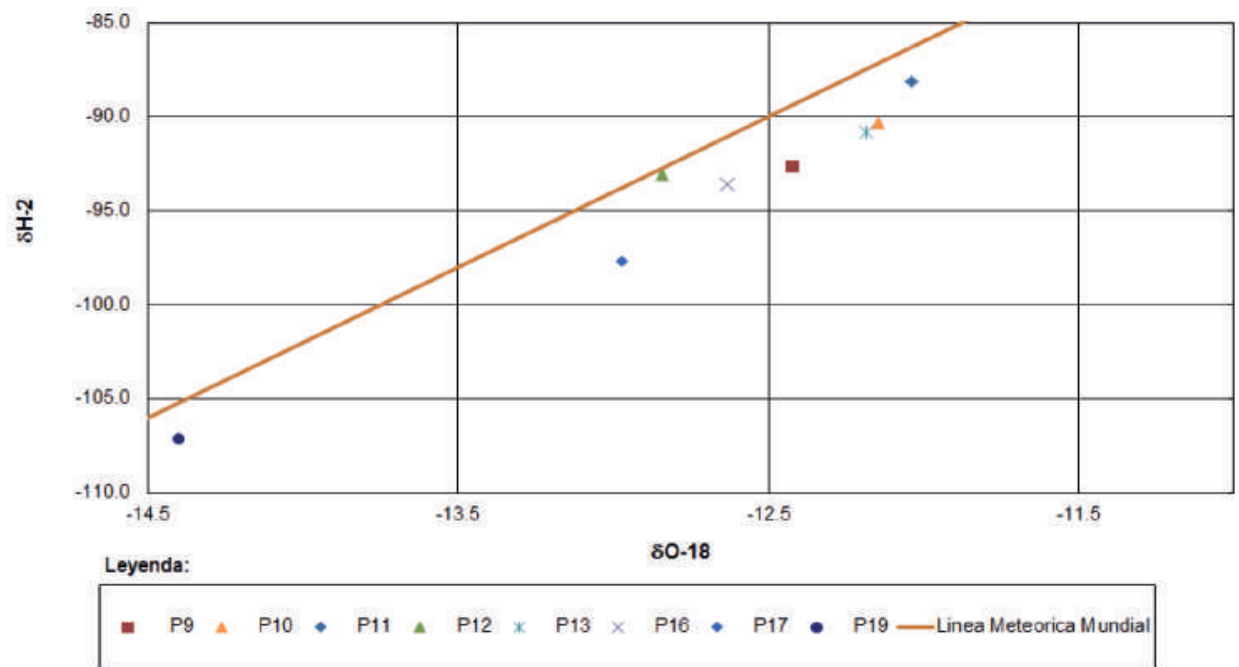

Figura 7. Análisis de $\delta O-18$ y $\delta H-2-$ Acuifero Pampas Lanchas.

d. Se realizó la caracterización hidrogeoquímica e isotópica mediante las siguientes evaluaciones:

- Evaluación geológica del área de estudio: zona de trasvase (lagunas), ríos Ica (afluentes Tambo y Santiago) y Pisco, manantiales y acuíferos (Valle Ica, Pampas Villacurí y Lanchas).

- Evolución hidrogeoquímica: diagramas Piper.

- Dinámica del sistema acuífero: oxígeno 18 y deuterio $2 \mathrm{H}$ y tritio $3 \mathrm{H}$.

- Zonas de recarga, descarga.

- Edad del agua ${ }^{14} \mathrm{C}$ y ${ }^{13} \mathrm{C}$ y tritio ${ }^{3} \mathrm{H}$.

- Deducción roca fuente: relaciones iónicas.

A fin de perfeccionar el modelo hidrogeológico, estableciendo políticas adecuadas para la gestión y protección del agua subterránea, se comprobó el sistema de recarga mostrado en la figura 8. 


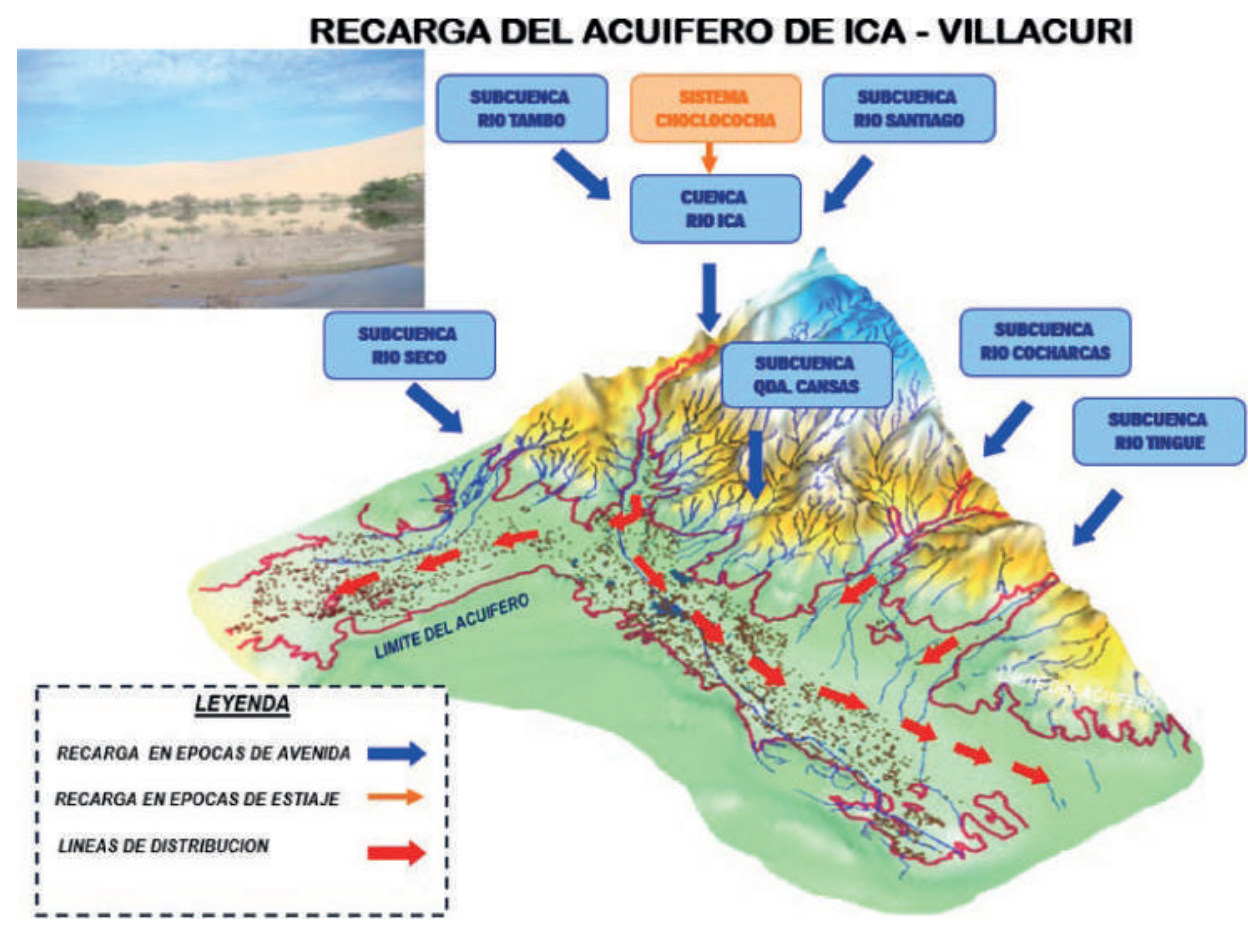

Figura 8. Mecanismo de recarga de los acuiferos de Ica y Villacurí.

Para la caracterización hidrogeoquímica del agua subterránea de los acuíferos, se ha considerado por su importancia de ser mayoritarios, el calcio $\left(\mathrm{Ca}^{++}\right)$, magnesio $\left(\mathrm{Mg}^{++}\right)$, sodio $\left(\mathrm{Na}^{+}\right)$, potasio $\left(\mathrm{K}^{+}\right)$, bicarbonatos $\left(\mathrm{HCO}_{3}^{-}\right)$, sulfatos $\left(\mathrm{SO}_{4}{ }^{-}\right)$, cloruros $(\mathrm{Cl}-)$, y como parámetros de campo, la conductividad eléctrica $(\mathrm{CE})$, la temperatura $\left(\mathrm{T}^{\circ}\right)$, sólidos totales disueltos y el $\mathrm{pH}$, usando como bases de caracterización los estándares de calidad de agua establecidos por el estado peruano y en el ámbito internacional, el de la Organización Mundial de la Salud (OMS).

En el tratamiento de los datos obtenidos, relacionados con el aseguramiento de la calidad de los análisis químicos, se utilizó la relación del balance iónico mediante el cálculo del porcentaje de error, aceptando como máximo un valor de $10 \%$, de tal manera que las muestras que rebasaron este límite no fueron consideradas para fines de interpretación.

Se usó el software AQUACHEM para realizar los gráficos de Piper, para lo cual se conformó una base de 615 análisis. Las figuras 9, 10 y 11, muestran los diagramas de Piper correspondientes a la evolución hidrogeoquímica de los acuíferos del Valle Ica, Villacurí y Lanchas, respectivamente. 

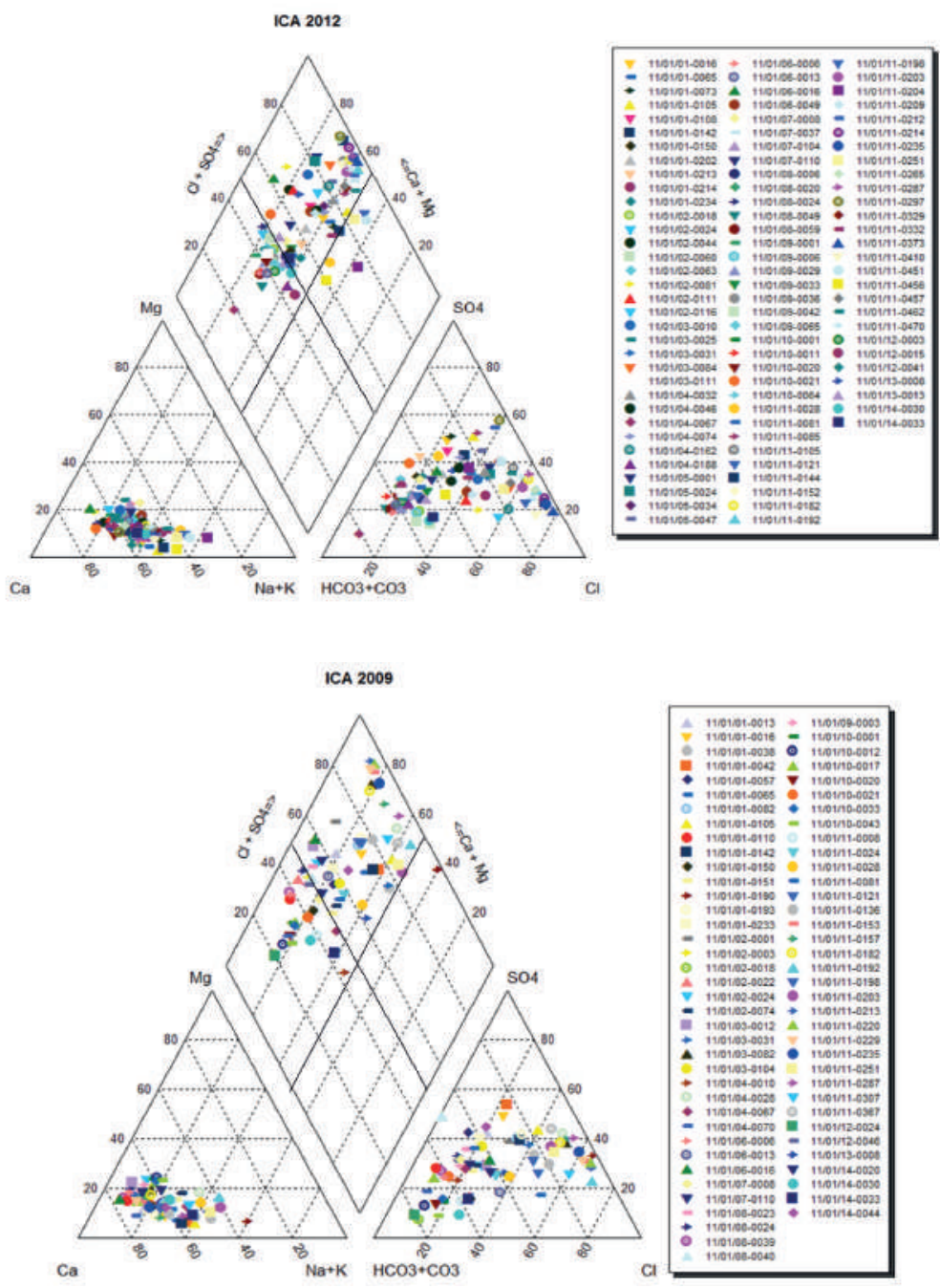

Figura 9. Evolución hidrogeoquimica: diagramas de Piper-Acuifero del Valle de Ica.

Análisis: Se determina la agrupación en el rombo superior correspondiente a las familias clorurada cálcica y sulfatada cálcica, presentes en todo el valle; especialmente en los distritos de la zona II (Ica, Pachacútec, Los Aquijes), seguida de la bicarbonatada cálcica ubicada en el rombo de la izquierda, presentes en la zona I (distritos de Los Molinos, La Tinguiña, Guadalupe, San Juan Bautista y Subtanjalla) y de la sulfatada sódica y clorurada sódica en el rombo derecho, correspondiendo a aguas con problemas de degradación en su calidad, pudiendo deberse a contaminación por sales, ubicadas predominantemente en los distritos de Santiago y Ocucaje (zona III). 

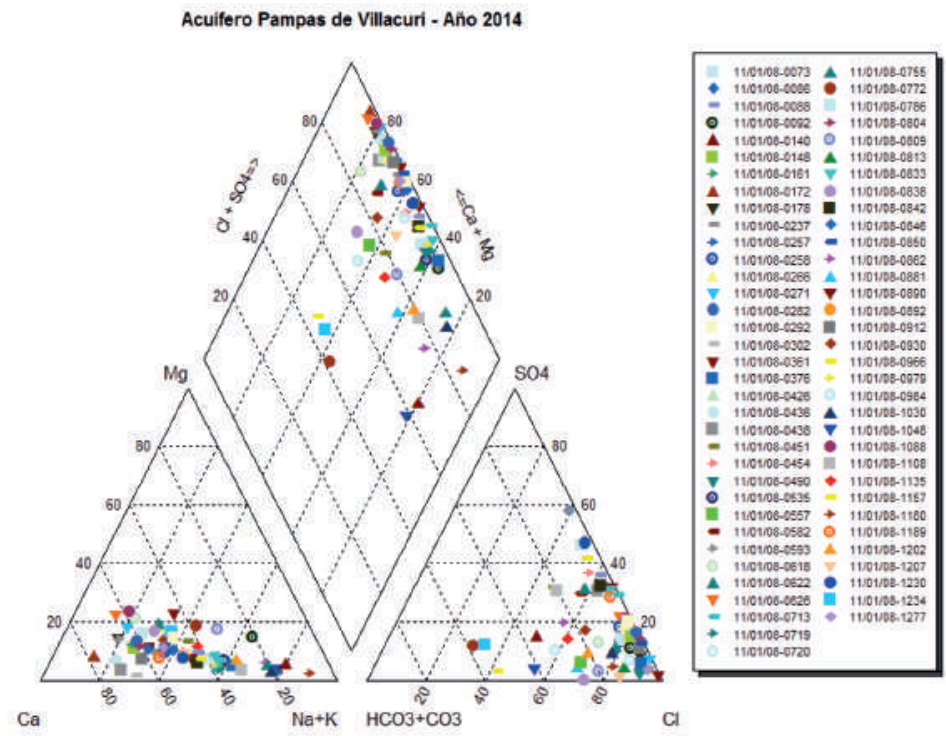

$\mathrm{Ca}$
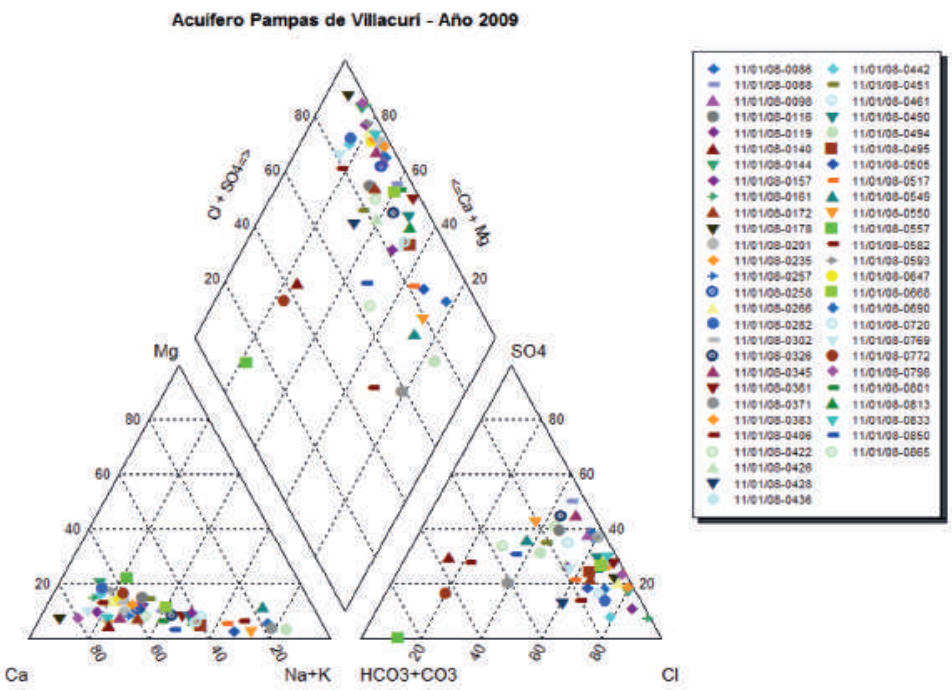

Figura 10. Evolución hidrogeoquímica: diagramas de Piper-Acuifero Pampas Villacurí.

Análisis: En general, las aguas presentes en este acuífero, corresponden a las familias cloruradas y sulfatadas cálcicas y/o magnésicas, y aguas cloruradas y sulfatadas sódicas. Debido a la sobreexplotación, se ha dado la degradación de la calidad del agua subterránea. 

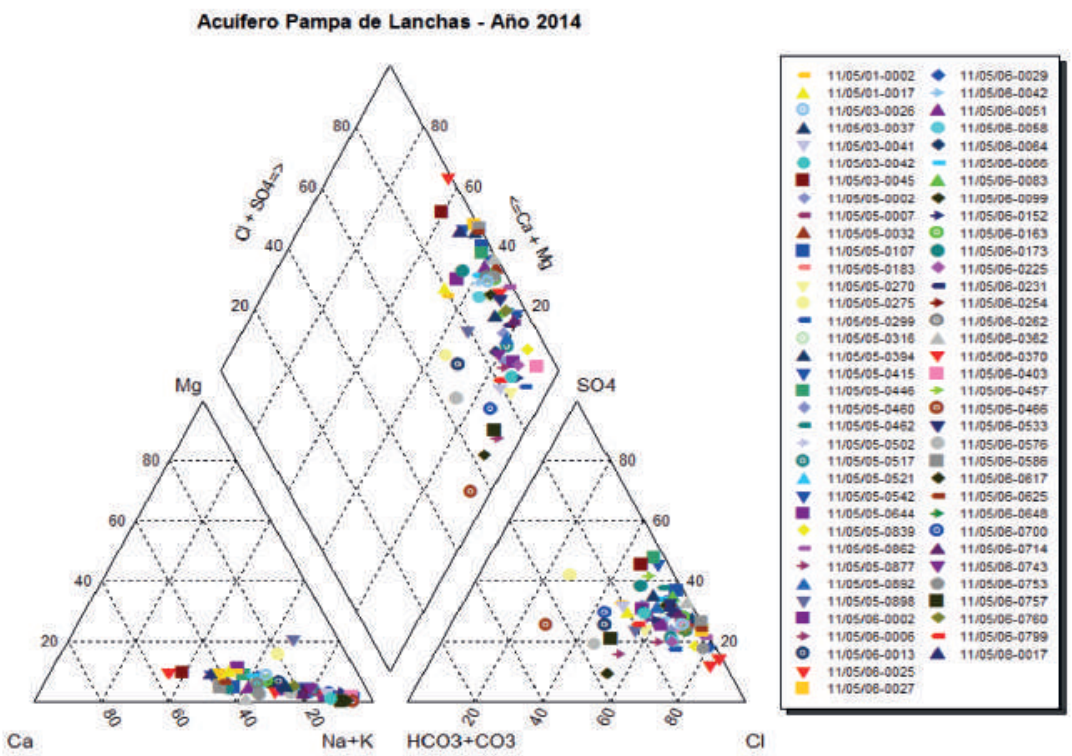

$\mathrm{Ca}$
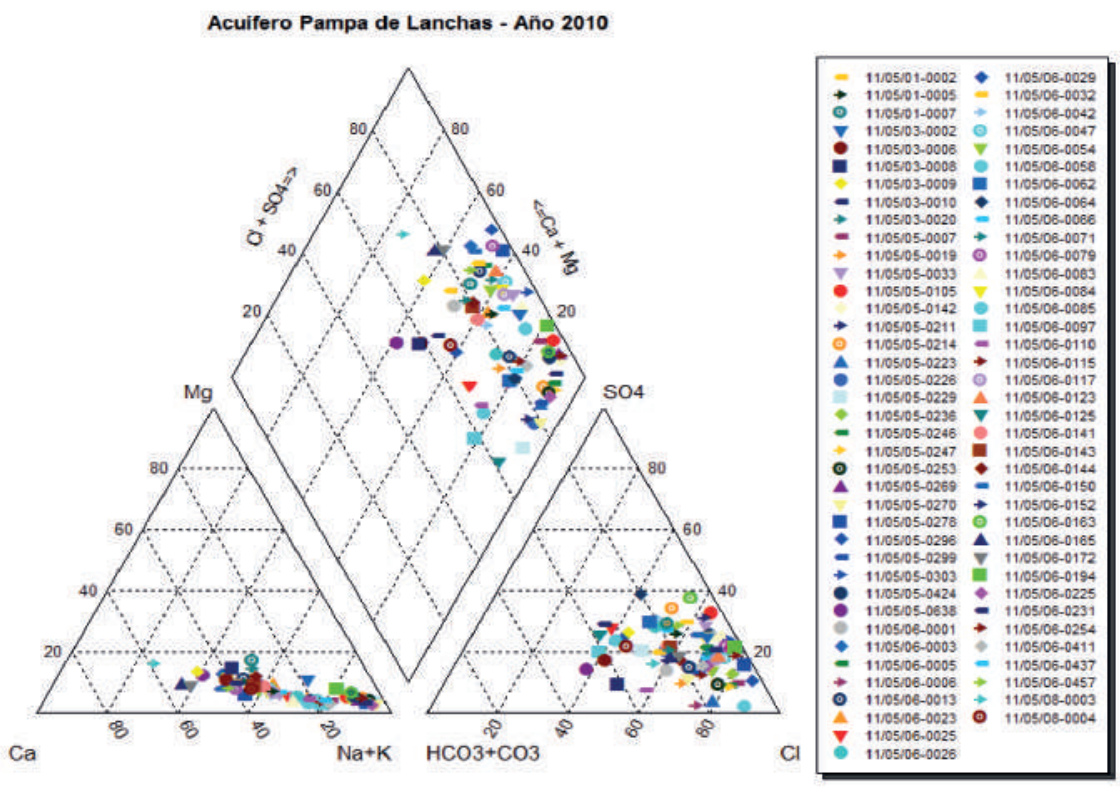

Figura 11. Evolución hidrogeoquimica: diagramas de Piper-Acuifero Pampas Lanchas.

Análisis: Las aguas de este acuífero en general son sulfatadas y cloruradas sódicas. Cabe indicar que estas aguas presentan altos valores de conductividad eléctrica y alta concentración de cloruros (degradación de la calidad del agua). 


\section{Conclusiones}

1. La conductividad eléctrica del agua subterránea en el acuífero de Ica varía con una tendencia al incremento de aguas arriba hacia aguas abajo, siguiendo aproximadamente el sentido preferencial del flujo subterráneo, en razón de que dicho flujo se represa por el estrechamiento del acuífero debido a la presencia de afloramientos rocosos.

2. En lo que corresponde al acuífero de Villacurí, la conductividad eléctrica varía desde la entrada de la Pampa de Villacurí hasta la salida hacia Pampa de Lanchas. Sin embargo, en el trayecto del flujo subterráneo se distinguen tres zonas con alta concentración salina, atribuible a la presencia de zonas con mayor velocidad de descenso del nivel de la napa.

3. De acuerdo al monitoreo de los pozos que conforman la red hidrogeoquímica de Ica y Villacurí, se observa que la evolución de la salinidad de las aguas en función de la conductividad eléctrica es variable (ascensos y descensos), presentándose los mayores incrementos en Villacurí, debido a la limitada recarga local del acuífero por ausencia de fuentes de agua superficial para riego.

4. En relación a las familias hidrogeoquímicas, se establece que en el valle de Ica predominan las familias de la clorurada cálcica, seguida de la bicarbonatada cálcica. En Villacurí las familias más predominantes corresponden a las bicarbonatadas cálcicasódicas y la clorurada cálcica.

5. En cuanto a las características hidrogeoquímicas del acuífero Pampa de Lanchas, la conductividad eléctrica, en el área de inventario presenta valores que corresponden a aguas de baja a alta mineralización. Prevalece la familia clorurada sódica, aunque se observa en menor proporción la clorurada cálcica y la bicarbonatada cálcica.

6. Según el análisis, se ha identificado como el problema central la sobreexplotación de los acuíferos del área del proyecto, evidenciado por el descenso continuo de los niveles de la napa freática, hecho que viene afectando reservas permanentes (no renovables) de agua subterránea y, en algunos casos, la calidad de la misma como en los acuíferos de Villacurí.

\section{Recomendaciones}

a. Es necesario simular el funcionamiento hidráulico del acuífero para varios escenarios y volúmenes de explotación y recarga, presentando los resultados en cuadros, gráficos y mapas, de cuya interpretación se determinará el volumen explotable y sostenible, que representa la oferta de agua subterránea del acuífero. 
b. Se debe promover el desarrollo de proyectos de recarga natural de carácter local (época de avenidas) y de recarga artificial (considerando trasvase de agua superficial de otras cuencas).

c. El trasvase de agua superficial permite emplear en forma conjunta el agua superficial y subterránea, disminuyendo el volumen de explotación de aguas subterráneas por reemplazo con agua superficial empleada directamente.

d. Se debe promover la formulación y desarrollo de proyectos de recarga de carácter local (rehabilitación y mantenimiento de canales sin revestir; utilización de pozas existentes y por construir).

e. Es necesario coordinar con las juntas de usuarios de riego y con las empresas agrícolas interesadas en la recarga local para la distribución de agua superficial en épocas de avenidas.

f. La recarga de los acuíferos se determinará en base a la evaluación de las fuentes naturales de alimentación, cuantificando la infiltración a partir del río, canales sin revestir y áreas bajo riego, así como los escurrimientos subterráneos que se producen por interconexión hidráulica.

\section{Referencias}

Aguilar, G. (2004). Diagnóstico de la oferta hidrica de los acuiferos del valle de Ica y Pampas de Villacurí. PETACC.

Asesores Técnicos Asociados S.A. (2002). Plan de gestión de la oferta de agua en las cuencas del ámbito del Proyecto Tambo-Ccaracocha. Aspectos hidrogeológicos.

Autoridad Nacional del Agua (2012). Plan de gestión del acuifero del valle de Ica y de las Pampas de Villacurí y Lanchas.

Autoridad Nacional del Agua (2011). Afianzamiento hidrico en la cuenca del río Pisco a nivel de factibilidad.

Autoridad Nacional del Agua (2010). Inventario de fuentes de agua subterránea Pampas de Lanchas.

De PAz, R. (2005). Modelo del acuifero de Ica-Villacurí.

Ingemmet (2010). Hidrogeología de la cuenca del río Ica.

InRENA (2006). Estudio hidrogeológico del valle de Pisco.

InRENA (1997). Inventario y monitoreo de las fuentes de agua subterránea en el Valle Ica y Pampas de Villacurí. 